\title{
BIRATIONAL TRANSFORMATIONS IN 4-SPACE AND 5-SPACE
}

\section{A. R. WILliams}

The purpose of this paper is to describe certain Cremona transformations in 4-space and 5-space, especially the former. The transformations in question are interesting on account of analogies with transformations in ordinary space, because they can be simply expressed and effectively studied by the use of equations, as well as synthetically, and because of the classic nature of the loci involved. A rational quartic curve in $S_{4}$ and a Veronese surface in $S_{5}$ are of special importance.

1. The $T_{2-4}$ in $S_{4}$. Through $C_{1}^{4}$, a rational quartic curve in $S_{4}$, pass $\infty^{5}$ hyperquadrics. Three of these intersect in a residual curve of order 4 , also rational, that meets $C_{1}^{4}$ six times. Therefore through the $C_{1}^{4}$ and a general point $O$ of $S_{4}$ pass $\infty^{4}$ hyperquadrics, four of which have one variable point in common. Thus the $\infty^{4}$ hyperquadrics, which may be conveniently called the system $(\phi)$, may be put in 1:1 correspondence with the hyperplanes of the same or another $S_{4}$. The $C_{1}{ }^{4}$ and the point $O$ constitute the fundamental points of the first space, or the first fundamental system. Three $\phi$ 's have in common a residual rational quartic $c$ to which corresponds a line in the second space. To intersections of $c$ with a hyperplane correspond intersections of the line with a hypersurface of the system $\left(\phi^{\prime}\right)$. The latter are of order 4 , and the transformation is described by the symbol $T_{2-4}$.

Let the rational quartic $C_{1}^{4}$ be given by $x_{0}: x_{1}: x_{2}: x_{3}: x_{4}=t^{4}: t^{3}: t^{2}: t: 1$. The determinants of the matrix

$$
\left\|\begin{array}{llll}
x_{0} & x_{1} & x_{2} & x_{3} \\
x_{1} & x_{2} & x_{3} & x_{4}
\end{array}\right\|
$$

equated to zero give six linearly independent hyperquadrics containing the curve. Through a general point $O\left(y_{0}: y_{1}: y_{2}: y_{3}: y_{4}\right)$ of $S_{4}$ pass $\infty^{4}$ hyperquadrics that contain the curve. One of them is a cone with vertex at $O$. Its equation is the sum of the six products formed from the above matrix $\left(x_{0} x_{2}-x_{1}^{2}\right)\left(y_{2} y_{4}-y_{3}^{2}\right)+\left(x_{0} x_{3}-x_{1} x_{2}\right)\left(y_{2} y_{3}-y_{1} y_{4}\right)$ $+\cdots=0$. We may, however, define the transformation thus:

$$
\begin{gathered}
x_{0}^{\prime}: x_{1}^{\prime}: x_{2}^{\prime}: x_{3}^{\prime}: x_{4}^{\prime}=\left(x_{0} x_{2}-x_{1}^{2}\right):\left(x_{0} x_{3}-x_{1} x_{2}\right):\left(x_{0} x_{4}-x_{1} x_{3}\right): \\
\left(x_{1} x_{4}-x_{2} x_{3}\right):\left(x_{2} x_{4}-x_{3}^{2}\right) .
\end{gathered}
$$


This is to take for $O$ the point $(0: 0: 1: 0: 0)$. It is, to be sure, the intersection of the osculating planes to the curve at $(0: 0: 0: 0: 1)$ and $(1: 0: 0: 0: 0)$. But that does not render the transformation any less general. Equations (1) can be solved giving

$$
\begin{aligned}
& x_{0}: x_{1}: x_{2}: x_{3}: x_{4}=x_{2}^{\prime 2}\left(x_{0}^{\prime} x_{2}^{\prime}-x_{1}^{\prime 2}\right)-x_{0}^{\prime} x_{2}^{\prime}\left(x_{0}^{\prime} x_{4}^{\prime}-x_{1}^{\prime} x_{3}^{\prime}\right): \\
& \quad x_{1}^{\prime} x_{2}^{\prime}\left(x_{0}^{\prime} x_{4}^{\prime}-x_{1}^{\prime} x_{3}^{\prime}\right)+x_{0}^{\prime} x_{2}^{\prime 2} x_{3}^{\prime}: x_{0}^{\prime} x_{2}^{\prime 2} x_{4}^{\prime}-\left(x_{0}^{\prime} x_{4}^{\prime}-x_{1}^{\prime} x_{3}^{\prime}\right)^{2}: \\
& \quad x_{2}^{\prime} x_{3}^{\prime}\left(x_{0}^{\prime} x_{4}^{\prime}-x_{1}^{\prime} x_{3}^{\prime}\right)+x_{1}^{\prime} x_{2}^{\prime 2} x_{4}^{\prime}: x_{2}^{\prime}{ }^{2}\left(x_{2}^{\prime} x_{4}^{\prime}-x_{3}^{\prime 2}\right) \\
& -x_{2}^{\prime} x_{4}^{\prime}\left(x_{0}^{\prime} x_{1}^{\prime}-x_{1}^{\prime} x_{3}^{\prime}\right) .
\end{aligned}
$$

Since the expressions on the right of (2), except the third, contain the factor $x_{2}^{\prime}$ it follows that to the point $(0: 0: 1: 0: 0)$ corresponds the hyperplane $x_{2}^{\prime}=0$. It appears also from (2) that the quadric surface defined by $x_{2}^{\prime}=0, x_{0}^{\prime} x_{4}^{\prime}-x_{1}^{\prime} x_{3}^{\prime}=0$ lies doubly on the quartic homaloids, the system $\left(\phi^{\prime}\right)$. The jacobian of the expressions on the right of (1) consists of two factors, $x_{0} x_{4}-x_{1} x_{3}$ and

$$
\left|\begin{array}{lll}
x_{0} & x_{1} & x_{2} \\
x_{1} & x_{2} & x_{3} \\
x_{2} & x_{3} & x_{4}
\end{array}\right| .
$$

These two equated to zero give the unique hypercone of the system $(\phi)$ whose vertex is at $O(0: 0: 1: 0: 0)$ and the cubic hypersurface which is the locus of chords of $C_{1}^{4}$. They constitute the first principal system, that is, the loci in the first space that correspond to the fundamental points in the second space. It is seen immediately from equations (1) that to any point on the hypercone $x_{0} x_{4}-x_{1} x_{3}=0$ corresponds a point on the quadric surface $F_{2}^{\prime 2}$, that is, $x_{2}^{\prime}=0, x_{0}^{\prime} x_{4}^{\prime}-x_{1}^{\prime} x_{3}^{\prime}=0$. To the cubic hypersurface, locus of the chords of $C_{1}{ }^{4}$, corresponds a rational quartic surface $F_{2}^{\prime 4}$ whose parametric representation will be obtained shortly. To $C_{1}^{4}$ corresponds a sextic hypersurface in the second space, since $C_{1}^{4}$ is met six times by the residual curve of intersection of any three $\phi$ 's. In fact, the jacobian of the expressions on the right of (2) is of order 15 and consists of the factor $x_{2}^{\prime 3}$ and a sextic factor taken twice, the latter corresponding to $C_{1}^{4}$. We can obtain this sextic hypersurface by simply substituting from (2) into (1). We find $x_{0}^{\prime}: x_{1}^{\prime}: x_{2}^{\prime}: x_{3}^{\prime}: x_{4}^{\prime}=x_{0}^{\prime} G^{\prime}: x_{1}^{\prime} G^{\prime}: x_{2}^{\prime} G^{\prime}: x_{3}^{\prime} G^{\prime}: x_{4}^{\prime} G^{\prime}$, where $G^{\prime}$ is of degree 7 , and consists of $x_{2}^{\prime}$ and the sextic factor $x_{2}^{\prime 3}\left(x_{0}^{\prime} x_{2}^{\prime} x_{4}^{\prime}-x_{0}^{\prime} x_{3}^{\prime 2}-x_{1}^{\prime 2} x_{4}^{\prime}\right)-x_{2}^{\prime 2}\left(x_{0}^{\prime} x_{4}^{\prime}-x_{1}^{\prime} x_{3}^{\prime}\right)\left(2 x_{0}^{\prime} x_{4}^{\prime}+x_{1}^{\prime} x_{3}^{\prime}\right)$ $+\left(x_{0}^{\prime} x_{4}^{\prime}-x_{1}^{\prime} x_{3}^{\prime}\right)^{3}$. The latter equated to zero gives the sextic hypersurface in question. The quadric surface $F_{2}^{\prime 2}$, that is, $x_{2}^{\prime}=0$, $x_{0}^{\prime} x_{4}^{\prime}-x_{1}^{\prime} x_{3}^{\prime}=0$, is evidently a triple surface on it. The sextic hypersurface is composed of $\infty^{1}$ planes that correspond to the points 
of $C_{1}{ }^{4}$. All points of a chord of $C_{1}{ }^{4}$ in the first space present the same condition to $(\phi)$, and any $\phi$ that contains a general point of such a chord contains all of it. Hence to each chord of $C_{1}^{4}$ corresponds a single (fundamental) point in the second space. In fact we note that a general point of the line joining the points whose parameters are $t_{1}$ and $t_{2}$ has the coordinates $\left(t_{1}^{4}+k t_{2}^{4}: t_{1}^{3}+k t_{2}{ }^{3}: t_{1}^{2}+k t_{2}^{2}: t_{1}+k t_{2}: 1+k\right)$. Substituting in (1) we have $x_{0}^{\prime}: x_{1}^{\prime}: x_{2}^{\prime}: x_{3}^{\prime}: x_{4}^{\prime}=t_{1}^{2} t_{2}^{2}: t_{1} t_{2}\left(t_{1}+t_{2}\right)$ : $t_{1}^{2}+t_{1} t_{2}+t_{2}^{2}: t_{1}+t_{2}: 1$. Setting $t_{1} t_{2}=l / n, t_{1}+t_{2}=m / n$, we have the ratios $l^{2}: l m: m^{2}-l n: m n: n^{2}$. Hence the surface is rational of order 4 . Its hyperplane sections are rational quartic curves whose images in the plane $l: m: n$ are conics. This quartic surface lies simply on the quartic homaloids $\left(\phi^{\prime}\right)$ and doubly on the sextic hypersurface that corresponds to the points of $C_{1}^{4}$. Therefore a quartic homaloid $\phi^{\prime}$ meets the sextic hypersurface in four planes which correspond to the points in which the corresponding hyperplane meets $C_{1}^{4}$, and in $F_{2}^{\prime 4}$ taken twice and in ${F_{2}^{\prime}}^{2}$ taken six times.

A general homaloid of the first space has the equation $a_{0}\left(x_{0} x_{2}-x_{1}^{2}\right)$ $+a_{1}\left(x_{0} x_{3}-x_{1} x_{2}\right)+a_{2}\left(x_{0} x_{4}-x_{1} x_{3}\right)+a_{3}\left(x_{1} x_{4}-x_{2} x_{3}\right)+a_{4}\left(x_{2} x_{4}-x_{3}^{2}\right)=0$. It contains the chord whose parameters are $t_{1}$ and $t_{2}$ if $a_{0} t_{1}^{2} t_{2}{ }^{2}$ $+a_{1} t_{1} t_{2}\left(t_{1}+t_{2}\right)+a_{2}\left(t_{1}^{2}+t_{1} t_{2}+t_{2}^{2}\right)+a_{3}\left(t_{1}+t_{2}\right)+a_{4}=0$. The corresponding hyperplane meets the quartic surface in a rational quartic whose image in the plane $l: m: n$ has the equation $a_{0} l^{2}+a_{1} l m+a_{2}\left(m^{2}-l n\right)$ $+a_{3} m n+a_{4} n^{2}=0$. The conic in $l: m: n$ may be two intersecting lines or a perfect square. Correspondingly there are $\infty^{3}$ hyperplanes that meet the surface in two conics having a common point, and $\infty^{1}$ hyperplanes that are tangent to it along a conic. The last system is of order 4 in the sense that 4 such hyperplanes pass through a general point of $S_{4}$. Fixing $t_{1}$ we see that to the chords projecting $C_{1}^{4}$ from the point $t_{1}$ corresponds a conic on the quartic surface $F_{2}^{\prime 4}$, which is represented in the plane $l: m: n$ by $l-t_{1} m+t_{1}^{2} n=0$. There are $\infty^{1} \phi$ 's which are hypercones having the point $t_{1}$ for vertex. To each of them corresponds a hyperplane in the second space that meets ${F_{2}^{\prime}}^{\prime}$ in this fixed conic and one variable conic that meets the fixed conic in one point.

The hypercone $x_{0} x_{4}-x_{1} x_{3}=0$ in the first space deserves further mention. It consists of the $\infty^{1}$ trisecant planes of $C_{1}^{4}$ that pass through $O(0: 0: 1: 0: 0)$. Thus the members of $(\phi)$ meet such a plane in the conics of a pencil. To each conic corresponds a point; and to the pencil of conics corresponds a line, since each $\phi$ in the first space contains one conic of the pencil. As noted above, these lines constitute the quadric surface ${F_{2}^{\prime}}^{2}$ which is double on the quartic homaloids in the second space, since its points correspond to curves of order 2. Three associated points of $C_{1}^{4}$ whose plane contains $(0: 0: 1: 0: 0)$ have 
the parameters $t, \omega t, \omega^{2} t$. The three degenerate conics of the pencil determined by these four points give three points, one of which is $\left(t^{4}:-t^{3}: 0:-t: 1\right)$, which lie on the generator of $F_{2}^{\prime 2}$ corresponding to the plane as a whole and on the rational quartic curve common to $F_{2}^{\prime 2}$ and $F_{2}^{\prime 4}$.

2. Special forms of the transformation. We may allow $O$ to approach a limiting position on $C_{1}^{4}$, or place $O$ on a chord or tangent to $C_{1}^{4}$, whereupon that chord or tangent becomes part of the curve common to $(\phi)$. If $O$ approaches a limiting position on $C_{1}^{4}$, the members of $(\phi)$ acquire a common tangent plane $e$ (not hyperplane) defined by the tangent to $C_{1}{ }^{4}$ and the direction of approach. The $\infty^{1}$ hyperplanes through $e$ meet $C_{1}^{4}$ in two associated points $P_{1}$ and $P_{2}$, and the $\infty^{1}$ planes determined by these pairs and the limiting position of $O$ constitute a hypercone, to which corresponds in the manner just described a quadric surface of fundamental points $F_{2}^{\prime 2}$. The variable intersection of any three $\phi$ 's passes through $O$ and meets $C_{1}{ }^{4}$ five times. Therefore to $O$ corresponds a hyperplane, and to the general points of $C_{1}^{4}$ correspond planes forming a quintic hypersurface. To the chords of $C_{1}^{4}$ corresponds again a quartic surface of fundamental point $F_{2}^{\prime 4}$. If $O$ approaches $(0: 0: 0: 0: 1)$ along the line joining the latter to ${ }^{\mathrm{s}}(0: 1: 1: d: e)$, the homaloids acquire the common tangent plane $x_{0}=x_{1}-x_{2}=0$; and we have the transformation $x_{0}^{\prime}: x_{1}^{\prime}: x_{2}^{\prime}: x_{3}^{\prime}: x_{4}^{\prime}$ $=\left(x_{0} x_{2}-x_{1}^{2}\right):\left(x_{0} x_{3}-x_{1} x_{2}\right):\left(x_{1} x_{3}-x_{2}^{2}\right):\left(x_{0} x_{4}-x_{1} x_{3}\right):\left[\left(x_{1} x_{4}-x_{2} x_{3}\right)\right.$ $\left.-\left(x_{2} x_{4}-x_{3}^{2}\right)\right]$. If we form the jacobian of the expressions on the right, the equation of the hypercone corresponding to $F_{2}^{\prime 2}$ is seen to be $x_{0} x_{3}-x_{0} x_{2}+x_{1}^{2}-x_{1} x_{2}=0$. Two associated points on $C_{1}^{4}$ have the parameters $t$ and $t /(t-1)$. That is, the plane determined by them and $(0: 0: 0: 0: 1)$ lies in the hypercone just mentioned, and the latter consists of such planes. Two chords joining (0:0:0:0:1) to a pair of associated points give the same point on $x_{0}^{\prime}=x_{1}^{\prime}=x_{2}^{\prime}=0$, which is, therefore, a double line on the quartic surface $F_{2}^{\prime 4}$ and a generator of the second regulus of $F_{2}^{\prime 2}$. To the $\infty^{1}$ chords joining pairs of associated points corresponds a conic lying in the plane $x_{0}^{\prime}-x_{1}^{\prime}=0$, $x_{1}^{\prime}-x_{2}^{\prime}-x_{3}^{\prime}=0$. This conic and the line $x_{0}^{\prime}=x_{1}^{\prime}=x_{2}^{\prime}=0$ constitute the intersection of $F_{2}^{\prime 2}$ and $F_{2}^{\prime 4}$. Solving the equations defining the transformation for $x_{0}: x_{1}: x_{2}: x_{3}: x_{4}$, we find that to $(0: 0: 0: 0: 1)$ corresponds the hyperplane $x_{0}^{\prime}-x_{1}^{\prime}=0$; and the quadric $F_{2}^{\prime 2}$ is found to be $x_{0}^{\prime}-x_{1}^{\prime}=0, x_{0}^{\prime} x_{4}^{\prime}-x_{2}^{\prime} x_{3}^{\prime}=0$, and to be double on the quartic homaloids $\left(\phi^{\prime}\right)$.

More interesting is the case when $O$ is on a chord of $C_{1}^{4}$, that is, when the system $(\phi)$ consists of the $\infty^{4}$ hyperquadrics containing $C_{1}^{4}$ and the chord. Then the residual intersection of three $\phi$ 's is a cubic 
which meets the chord once and $C_{1}^{4}$ four times. Hence the transformation is a $T_{2-3}$. The $\infty^{1}$ planes determined by the chord and the points of $C_{1}{ }^{4}$ constitute a hyperquadric cone of the second species; that is, every point of the chord is a vertex. Such a plane is cut by the members of $(\phi)$ in the lines of a pencil with center on $C_{1}^{4}$. To each such line corresponds a point, and to the plane as a whole corresponds a line. To the totality of these lines, that is, to the hyperquadric cone, corresponds a quadric surface $F_{2}^{\prime 2}$, which, however, is a simple surface on the cubic homaloids $\left(\phi^{\prime}\right)$ since its points correspond to straight lines. To the chords of $C_{1}{ }^{4}$ corresponds a cubic surface. For two $\phi$ 's have three variable chords of $C_{1}{ }^{4}$ in common. To the fixed chord $A_{1} A_{2}$ corresponds a hyperplane since it is met by the variable intersection of three $\phi$ 's in one point. To the individual points of $A_{1} A_{2}$ correspond planes which must, therefore, form an axial pencil. To the points of $C_{1}^{4}$ correspond planes forming a quartic hypersurface.

To express such a transformation algebraically we have the equations $x_{0}^{\prime}: x_{1}^{\prime}: x_{2}^{\prime}: x_{3}^{\prime}: x_{1}^{\prime}=\left(x_{0} x_{2}-x_{1}^{2}\right):\left(x_{0} x_{3}-x_{1} x_{2}\right):\left(x_{1} x_{3}-x_{2}^{2}\right)$ : $\left(x_{1} x_{4}-x_{2} x_{3}\right):\left(x_{2} x_{4}-x_{3}^{2}\right)$. The expressions on the right equated to zero represent five linearly independent hyperquadrics which contain $C_{1}^{4}$ and the chord $x_{1}=x_{2}=x_{3}=0$ joining the points $(0: 0: 0: 0: 1)$ and $(1: 0: 0: 0: 0)$ whose parameters are 0 and $\infty$. To the chord joining the points of $C_{1}^{4}$ whose parameters are $t_{1}$ and $t_{2}$ corresponds the point $t_{1}^{2} t_{2}^{2}: t_{1} t_{2}\left(t_{1}+t_{2}\right): t_{1} t_{2}: t_{1}+t_{2}: 1$. Writing as before $t_{1} t_{2}=l / n$, $t_{1}+t_{2}=m / n$, we have $x_{0}^{\prime}: x_{1}^{\prime}: x_{2}^{\prime}: x_{3}^{\prime}: x_{4}^{\prime}=l^{2}: \ln : \ln : m n: n^{2}$. The expressions on the right vanish for $l=n=0$. Therefore the surface corresponding to the chords of $C_{1}^{4}$ is a cubic $F_{2}^{\prime 3}$. Corresponding to the point $(0: 1: 0)$ of the plane $l: m: n$ is the line of the surface $x_{0}^{\prime}=x_{2}^{\prime}=x_{4}^{\prime}=0$. To the lines $l=0$ and $n=0$ of the same plane correspond the lines of the surface $x_{0}^{\prime}=x_{1}^{\prime}=x_{2}^{\prime}=0$, and $x_{2}^{\prime}=x_{3}^{\prime}=x_{4}^{\prime}=0$. It will be noted that these three lines constitute the intersection of the cubic surface with the hyperplane $x_{2}^{\prime}=0$, and that the second and third lie on the quadric surface $x_{2}^{\prime}=0, x_{0}^{\prime} x_{4}^{\prime}-x_{1}^{\prime} x_{3}^{\prime}=0$. This is the quadric surface $F_{2}^{\prime 2}$ that corresponds to the hypercone composed of the planes determined by the chord $A_{1} A_{2}$ and the points of $C_{1}^{4}$. And $x_{2}^{\prime}=0$ is the hyperplane that corresponds to the chord $A_{1} A_{2}$. For let $P(k: 0: 0: 0: 1)$ be a general point of that chord. By allowing a point $Q$ to approach $P$ in an arbitrary direction we find easily that the plane corresponding to $(k: 0: 0: 0: 1)$ is defined by $x_{2}^{\prime}=0, x_{0}^{\prime}-k x_{4}^{\prime}=0$. Hence to the chord as a whole corresponds the 3 -space $x_{2}^{\prime}=0$ made up of the pencil of planes whose axis is $x_{2}^{\prime}=x_{0}^{\prime}=x_{4}^{\prime}=0$. To the line joining $(k: 0: 0: 0: 1)$ and $\left(t^{4}: t^{3}: t^{2}: t: 1\right)$, a general point of $C_{1}^{4}$, corresponds the point $\left(k t: k: 0: t^{2}: t\right)$. All such points lie on the quadric surface 
$F_{2}^{\prime 2}$ defined by $x_{2}^{\prime}=0, x_{0}^{\prime} x_{4}^{\prime}-x_{1}^{\prime} x_{3}^{\prime}=0$. When $t$ is fixed and $k$ varies, we get the line $x_{2}^{\prime}=0, x_{0}^{\prime}-t x_{1}^{\prime}=0, x_{3}^{\prime}-t x_{4}^{\prime}=0$, which corresponds to the plane joining the chord and the point $t$. When $k$ is fixed and $t$ varies, we get a conic whose plane is defined by $x_{2}^{\prime}=0, x_{0}^{\prime}-k x_{4}^{\prime}=0$. Putting $t=0$ and $k=0$, we have the pair of intersecting lines $x_{0}^{\prime}=x_{2}^{\prime}=x_{3}^{\prime}=0$ and $x_{0}^{\prime}=x_{1}^{\prime}=x_{2}^{\prime}=0$, which correspond respectively to the plane determined by the chord and the tangent at $(0: 0: 0: 0: 1)$ and to the cubic cone projecting $C_{1}^{4}$ from the same point. These lines meet the axis $x_{0}^{\prime}=x_{2}^{\prime}=x_{4}^{\prime}=0$ and determine a plane of the pencil tangent to $F_{2}^{\prime 2}$. For the plane determined by the chord and the tangent at $(1: 0: 0: 0: 0)$ and for the cone projecting $C_{1}{ }^{4}$ from that point, we have the similar pair $x_{1}^{\prime}=x_{2}^{\prime}=x_{4}^{\prime}=0$ and $x_{2}^{\prime}=x_{3}^{\prime}=x_{4}^{\prime}=0$ which give the other plane of the axial pencil tangent to $F_{2}^{\prime 2}$. The quadric surface $F_{2}^{\prime 2}$ lies simply, and $F_{2}^{\prime 3}$ lies doubly, on the quartic hypersurface composed of planes that correspond to the points of $C_{1}^{4}$. They are both simple surfaces on the cubic homaloids $\left(\phi^{\prime}\right)$. It should be noted that $C_{1}{ }^{4}$ and the chord $A_{1} A_{2}$ constitute a quintic of genus 1 ; and it is well known that through a quintic of genus 1 pass $\infty^{4}$ hyperquadrics three of which have for residual intersection a cubic that meets the quintic five times. A Cremona transformation can be constructed on that basis.

3. The $T_{2-2}$ in $S_{5}$. Coming now to 5 -space, we see that there is a simple Cremona transformation which is quadratic both ways, and which therefore may be an involution. There are $\infty^{5}$ hyperquadrics which have a Veronese surface $F_{2}{ }^{4}$ in common. Three of them have a residual intersection which is also a Veronese surface, and which has a sextic curve of genus 1 in common with $F_{2}^{4}$. A fourth hyperquadric of the system meets the variable Veronese surface in a curve of order 8 consisting of the sextic and a conic $c$ which meets the sextic curve, and so $F_{2}{ }^{4}$, three times. Hence five hyperquadrics of the system have one variable point in common. We have thus a transformation in which the conic $c$ corresponds to a line, and which is therefore a $T_{2-2}$. The first principal system consists of the $\infty^{2}$ planes of the conics on $F_{2}{ }^{4}$. To each such plane corresponds a point. To the $\infty^{1}$ such planes passing through a point $P$ of $F_{2}^{4}$ correspond the points of a conic. For a hyperquadric $\phi$ contains two of them. In fact the tangent hyperplane to $\phi$ at $P$ meets $F_{2}^{4}$ in a quartic curve which consists of two of the conics lying on $F_{2}{ }^{4}$ and passing through $P$. The planes of these conics lie on $\phi$. Thus each $\phi$ contains $\infty^{1}$ planes of conics belonging to $F_{2}{ }^{4}$, and two $\phi$ 's have four such planes in common. Hence the surface of fundamental points in the second space is of order 4 and contains $\infty^{2}$ conics. It is therefore a Veronese surface $F_{2}^{\prime}{ }^{4}$. To the points of $F_{2}{ }^{4}$ 
correspond the $\infty^{2}$ planes of the conics on $F_{2}^{\prime 4}$. They constitute a cubic hypersurface, since it is met by a line in three points corresponding to the points in which the residual intersection (conic) of four $\phi$ 's meets $F_{2}{ }^{4}$.

This transformation in its involutory form may be expressed by a very simple system of equations. The Veronese surface $F_{2}^{4}$ is rational, and its $\infty^{5}$ hyperplane sections correspond to the conics of the plane of representation. Hence the points of $F_{2}^{4}$ are given by $x_{0}: x_{1}: x_{2}: x_{3}: x_{4}: x_{5}=l^{2}: \operatorname{lm}: \ln : m^{2}: m n: n^{2}$. Thus the minors of

$$
\left|\begin{array}{lll}
x_{0} & x_{1} & x_{2} \\
x_{1} & x_{3} & x_{4} \\
x_{2} & x_{4} & x_{5}
\end{array}\right|
$$

equated to zero give six linearly independent hyperquadrics containing $F_{2}^{4}$. The determinant itself equated to zero is the equation of the locus of the planes of the $\infty^{2}$ conics that lie on $F_{2}^{4}$. These planes contain all the chords of the surface, since through any two points of it passes one of the conics. This suggests the equations of transformation (1) $x_{0}^{\prime}: x_{1}^{\prime}: x_{2}^{\prime}: x_{3}^{\prime}: x_{4}^{\prime}: x_{5}^{\prime}=\left(x_{3} x_{5}-x_{4}^{2}\right):\left(x_{2} x_{4}-x_{1} x_{5}\right):\left(x_{1} x_{4}-x_{2} x_{3}\right)$ : $\left(x_{0} x_{5}-x_{2}^{2}\right):\left(x_{1} x_{2}-x_{0} x_{4}\right):\left(x_{0} x_{3}-x_{1}^{2}\right)$. The jacobian of the expressions on the right is of course the above determinant taken twice. If we substitute on the right for the unprimed letters the same functions of the primed letters we get $x_{0}^{\prime}: x_{1}^{\prime}: x_{2}^{\prime}: x_{3}^{\prime}: x_{4}^{\prime}: x_{5}^{\prime}=x_{0}^{\prime} G^{\prime}: x_{1}^{\prime} G^{\prime}: x_{2}^{\prime} G^{\prime}: x_{3}^{\prime} G^{\prime}$ : $x_{4}^{\prime} G^{\prime}: x_{5}^{\prime} G^{\prime}$, where $G^{\prime}$ is the above determinant in the primed letters, and $G^{\prime}=0$ gives the locus of points corresponding to the points of $F_{2}^{4}$. Thus we have a simple involution by which the points of $S_{5}$ are paired. The points of the Veronese surface $F_{2}^{4}$, however, correspond to the planes of its conics, and vice versa. This correspondence is double in the sense that if a point and plane of $F_{2}{ }^{4}$ correspond to each other by the direct transformation they do also by the reverse. In fact such a point and plane pair may be defined in the following way by the same three numbers. Let $a l+b m+c n=0$ be the equation of a line in the plane $l: m: n$ representing the Veronese surface. Three points on this line are $(0: c:-b),(c: 0:-a),(b:-a: 0)$. The corresponding points on $F_{2}{ }^{4}$ are $\left(0: 0: 0: c^{2}:-b c: b^{2}\right)$ and two others whose coordinates are similar combinations of the letters $c a$ and $a b$. These points are in the plane of the conic on $F_{2}^{4}$ which corresponds to $l a+m b+n c=0$. If we take a general point of their plane and apply equations (1) above we get $x_{0}^{\prime}: x_{1}^{\prime}: x_{2}^{\prime}: x_{3}^{\prime}: x_{4}^{\prime}: x_{5}^{\prime}=a^{2}: a b: a c: b^{2}: b c: c^{2}$, which is the point on the Veronese surface that corresponds to the point $a: b: c$ in the plane of representation.

University of CALIFornia 